\title{
La educación y los entornos virtuales de aprendizaje
}

\section{Education and virtual learning environments}

1 Jessica Mariela Carvajal Morales

Universidad Estatal de Milagro, Milagro, Ecuador.

jcarvajalm4@unemi.edu.ec

2 Daira Marizol Carvajal Morales Universidad Estatal de Milagro, Milagro, Ecuador.

dcarvajalm2@unemi.edu.ec

3 Javier Guaña Moya

https://orcid.org/0000-0002-4312-9440 Instituto Superior Tecnológico Japón, Quito, Ecuador.

eguana@itsjapon.edu.ec

4 Kelvin Antonio Mendoza Zambrano

Reybanpac, Rey Banano del Pacífico C.L., Quevedo, Ecuador.

kmendoza@favoritafc.com

Artículo de Investigación Científica y Tecnológica

Enviado: 10/12/2021

Revisado: 27/12/2021

Aceptado: 07/01/2022

Publicado:23/02/2022

DOI: https://doi.org/10.33262/ap.v4i1.2.186

Cítese: Carvajal Morales, J. M., Carvajal Morales, D. M., Guaña Moya, J., \& Mendoza Zambrano, K. A. (2022). La educación y los entornos virtuales de aprendizaje. AlfaPublicaciones, 4(1.2), 78-90. https://doi.org/10.33262/ap.v4i1.2.186

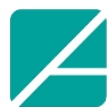

Ciencia

Digital

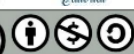

ALFA PUBLICACIONES, es una Revista Multidisciplinar, Trimestral, que se publicará en soporte electrónico tiene como misión contribuir a la formación de profesionales competentes con visión humanística y crítica que sean capaces de exponer sus resultados investigativos y científicos en la misma medida que se promueva mediante su intervención cambios positivos en la sociedad. https://alfapublicaciones.com

La revista es editada por la Editorial Ciencia Digital (Editorial de prestigio registrada en la Cámara Ecuatoriana de Libro con No de Afiliación 663) www.celibro.org.ec 


Palabras
claves:
entornos
virtuales de
aprendizaje
(EVA),
tecnologías de
la información y
la comunicación
(tic), proceso
educativo,
herramientas
educativas.

Keywords: virtual learning environments (VLE), information and communication

\section{Resumen}

Introducción: La incidencia de las Tecnologías de la Información y la Comunicación (TIC) sobre la sociedad del conocimiento ha originado importantes cambios, especialmente en la forma de transmitirlo y el diseño del contenido, con un gran efecto masivo y multiplicador, de tal manera que el sentido del conocimiento se ha profundizado en todos los niveles sociales, resaltando que el mayor impacto registrado ha recaído sobre los procesos educativos, siendo en las instituciones escolares donde la tecnología ha ejercido la mayor influencia, así como también en la labor del docente, llegando a formar parte de los procesos de enseñanza tradicionales. Objetivo: Analizar el impacto de la incorporación de los entornos virtuales de aprendizaje (EVA) a la educación. Metodología: Por medio de una metodología cualitativa basada en una extensa revisión bibliográfica se analiza el impacto de los EVA sobre la educación, los tipos de herramientas tecnológicas disponibles actualmente, las diversas maneras de aplicación y los aportes que han realizado las TIC sobre los procesos educativos. Resultados: La revisión de literatura permite deducir que la incorporación de las TIC a la educación por medio de los entornos virtuales de aprendizaje (EVA) se ha convertido en un proceso fundamental que en su concepción va más allá de la simple aplicación de herramientas tecnológicas que forman parte del sistema educativo, se refiere a una construcción didáctica y la forma cómo se construye y consolida un aprendizaje significativo basado en la tecnología, por lo que en un sentido estrictamente pedagógico se habla del uso tecnológico a la educación. Conclusiones: Las herramientas tecnológicas como los EVA han incrementado el nivel de significancia y concepción educativa al establecer modelos novedosos de comunicación y generando espacios de formación, información, debate y reflexión, traspasando las barreras de los procesos educativos tradicionales, convirtiéndose en valiosos recursos para el aprendizaje y formación de estudiantes con elevados niveles de competencias personales y profesionales.

\footnotetext{
Abstract

Introduction: The incidence of Information and Communication Technologies (ICT) on the knowledge society has caused important changes, especially in the way of transmitting it and the design of the content, with a great massive and multiplier effect, in such a way that the sense of knowledge has deepened at all social levels, highlighting
} 
technologies

(ICT),

educational

process,

educational

tools. that the greatest recorded impact has fallen on educational processes, being in school institutions where technology has exerted the greatest influence, as well as in the work of the teacher, coming to form part of traditional teaching processes. Objective: Analyze the impact of the incorporation of virtual learning environments (VLE) to education. Methodology: Through a qualitative methodology based on an extensive bibliographic review, the impact of VLEs on education, the types of technological tools currently available, the many ways of application and the contributions that ICTs have made on educational processes are analyzed. Results: The literature review allows us to deduce that the incorporation of ICT in education through virtual learning environments (VLE) has become a fundamental process that in its conception goes beyond the simple application of technological tools that are part of the educational system, refers to a didactic construction and the way in which significant learning based on technology is built and consolidated, so that in a strictly pedagogical sense we speak of the use of technology in education. Conclusions: Technological tools such as VLEs have increased the level of significance and educational conception by establishing innovative communication models and generating spaces for training, information, debate and reflection, crossing the barriers of traditional educational processes, becoming valuable resources for learning and training of students with elevated levels of personal and professional skills.

\section{Introducción}

Con el desarrollo y la difusión de las Tecnologías de la Información y la Comunicación (TIC), se comenzó a generar un gran interés en conocer cómo las herramientas tecnológicas benefician y mejoran los procesos educativos y fomentan el uso de la tecnología en las aulas, siendo los entornos virtuales de aprendizaje (EVA), también llamadas plataformas de aprendizaje en línea, sistemas de gestión de aprendizaje o sistemas de gestión de cursos, uno de los principales recursos que ofrecen las TIC (Herrera, 2017).

Los EVA corresponden a espacios virtuales que los educadores y los estudiantes pueden usar para presentar y compartir recursos y actividades e interactuar entre ellos. Estas plataformas, generalmente se pueden utilizar para impartir cursos en línea completos o como una herramienta de apoyo para cursos presenciales, proporcionando un medio para gestionar y comunicar la experiencia de aprendizaje prevista, así como también facilitar 
la participación de profesores y alumnos en la experiencia. Por tanto, el objetivo principal es facilitar, motivar y proporcionar experiencias de aprendizaje que van más allá del aula, generando impactos positivos en los diversos contextos educativos (Barker \& Gossman, 2013).

Es difícil definir exactamente cuánto tiempo han estado en uso los EVA como recurso educativo; por ejemplo, se tiene el caso de la Universidad Abierta del Reino Unido que ha empleado computadoras para el aprendizaje desde los años 70, sin embargo, en el año 2000 fue cuando se patentó el EVA, conocido como Blackboard, de forma comercial y basado en computadoras, registrando que para el 2010 más de 3700 instituciones educativas en más de 60 países ya usaban este software. También se tiene el Moodle, considerado el EVA más popular, surgió en 2001 y originalmente tenía la finalidad de ayudar a los educadores a crear cursos en línea con un enfoque en la interacción y la construcción colaborativa de contenido, para el 2011, Moodle tenía una base de usuarios en 212 países con más de 49 mil sitios activos en más de 75 idiomas (Underwood, 2009).

Actualmente la mayor parte de las instituciones educativas en el mundo cuentan con espacios virtuales y herramientas de apoyo basadas en Internet, por lo que esta invasión tecnológica ha originado cambios importantes, especialmente en los docentes, debido se han visto obligados a actualizar perfiles y adaptar métodos pedagógicos con la finalidad de adquirir habilidades y destrezas en el ámbito tecnológico, desarrollando de forma efectiva y fluida la aplicabilidad de las nuevas tecnologías en concordancia con los objetivos del proceso de enseñanza-aprendizaje (Romero, 2019).

Lo cierto es que gracias al empleo de las TIC y, particularmente los EVA, el proceso de enseñanza-aprendizaje encuentra oportunidades, fortalezas, debilidades y amenazas que pueden ser manejadas en función de potenciar los resultados, partiendo del dominio intencional de los aspectos metodológicos básicos (Vidal et al., 2008). En consecuencia, la metodología de la educación en entornos virtuales aporta cambios sustanciales que el instructor o docente, de todos los niveles educativos, debe conocer y adaptar para alcanzar un desempeño eficiente de los actores que intervienen en el proceso.

\section{Metodología}

Se realizó una revisión bibliográfica de los últimos años donde se incluyeron tesis, artículos científicos y de revisión publicadas en los repositorios y las revistas indexadas, aplicando una metodología cualitativa con la finalidad de analizar el impacto de los EVA sobre la educación, los tipos de herramientas tecnológicas disponibles actualmente, las diversas maneras de aplicación para finalmente determinar los aportes que han realizado las TIC sobre los procesos educativos. 


\section{Desarrollo}

Los entornos virtuales de aprendizaje (EVA) son sistemas en línea que comprenden una gama de herramientas para apoyar y gestionar el aprendizaje y tienen como objetivo ofrecer espacios que faciliten la diversificación de los métodos de enseñanza en los diferentes niveles educativos (Romero, 2019).

Autores como Green et al. (2008), se refieren a la gestión de cursos y los sistemas de gestión del aprendizaje como paquetes de software que permiten al educador o instructor impartir partes o un curso completo a través de un entorno basado en la web, lo cual determina que los EVA desempeñan un papel fundamental al apoyar la instrucción, permitiendo que los maestros seleccionen y utilicen diferentes recursos y aplicaciones para revisar el contenido del curso.

Por otra parte Dudeney \& Hockly (2007), presentan los EVA como plataformas basadas en la web en las que se pueden almacenar los contenidos del curso, permitiendo crear cursos en línea y que los estudiantes se inscriban en ellos, otorgando la posibilidad que dentro de los propios cursos se combinen varios recursos con elementos más interactivos, tal como pruebas y cuestionarios, dando facilidad a los profesores para que usen el chat con el fin de comunicarse con los estudiantes directamente o enviar un mensaje, y si desean interactuar con los alumnos, pueden emplear Skype o videoconferencias para organizar una reunión, constituyendo estas situaciones ejemplos de cómo se pueden vincular herramientas externas como el EVA a un repositorio.

Es bien conocido que desde la aparición de los equipos de computación las TIC se han incorporado a la educación como parte importante de los procesos inherentes a ella desde diversas realidades, dando lugar a una extensa gama de usos y aplicaciones, por lo que, partiendo de esta visión, se pueden identificar claramente algunas formas de esta incorporación que, en mayor y menor grado, han transformado los contextos de educación formal, destacando los siguientes:

- Incorporación a aulas específicas para ser empleadas como herramientas complementarias para el acceso, almacenamiento, manejo y presentación de la información.

- Intervención por medio de contenidos específicos dentro del propio proceso enseñanza-aprendizaje, con el objetivo fundamental de enseñar a manejar las herramientas tecnológicas, además del estudio del software específico e inclusive las características del hardware, temas que tienen vigencia en la actualidad.

- Incorporación a las aulas tradicionales como herramientas de apoyo del proceso de enseñanza-aprendizaje de contenidos específicos.

- Gracias a las características de fácil acceso y portabilidad puede ser distribuido rápida y fácilmente entre los estudiantes e interesados. 
- Conexión en red, generalmente por medio del Internet, ampliando las posibilidades espaciales y temporales de acceso a los contenidos programáticos de los diversos programas educativos (Sánchez \& Salvador, 2010).

Por otra parte, de acuerdo a lo señalado por Vidal et al. (2008), se han identificado tres modelos básicos relacionados con el empleo de la tecnología como apoyo para los procesos de enseñanza-aprendizaje, que son:

1. Aulas virtuales como herramientas que complementan cursos tradicionales presenciales. Este formato se viene implementando en los diversos niveles educativos, desde la educación primaria hasta la educación adulta, siendo el propósito fundamental el intercambio de información y material académico, así como también la realización de proyectos conjuntos, propiciando la interacción social (Moscosio, 2001).

2. Redes informáticas y medios de comunicación que facilitan las interacciones entre estudiantes, docentes y materiales. Modelo de gran influencia en los métodos educativos actuales, centrando el desarrollo del proceso en la implementación de metodologías colaborativas, como es el caso de las instituciones de educación a distancia que emplean la herramienta tecnológica para complementar la comunicación entre el instructor y el estudiante (GarcíaAretio et al., 2007).

3. Cursos en red. Metodología aplicada desde 1980, inicialmente en el contexto universitario en aulas y campus, que se ha generalizado de manera progresiva, encontrando que en la actualidad gran parte de las instituciones educativas incluyen recursos como, por ejemplo, las videoconferencias como soporte tecnológico central.

Señala Hernández (2017), que existe una real integración de las TIC a la educación, cuando se evidencia que el sistema educativo adquiere la capacidad de diseñar y aplicar un aprendizaje significativo, producto de las experiencias vivenciales y contenidos reflexivos, logrando una efectiva generación de conocimiento, tanto para el alumno como para el docente, circunstancia que se focaliza no solo en el aula, sino que en cada espacio y momento donde se evidencia un aprendizaje se postule la idea de alcanzar este logro de significancia.

En relación a los tipos de EVA, estos pueden ser de dos tipos, en primer lugar, se encuentra el software vendido por una empresa a una institución educativa, con el cual el docente sigue las actividades y recursos cargados y, por otro lado, el educador configura un EVA por medio del uso de un software libre, esta clasificación indica que el primer tipo está dirigido directamente a la institución, mientras que el segundo se dirige al docente (Walker \& White, 2013). Sin embargo, para que los docentes consideren la aplicación de un EVA puede llevar mucho tiempo, debido que tiene que existir un proceso 
previo de adaptación de las actividades del EVA al contenido del curso y brindar retroalimentación personalizada a los estudiantes, por lo que el entorno virtual dirigido por la institución termina siendo la primera opción; pero para aquellos a quienes les gusta crear sus propias tareas y jugar con aplicaciones tecnológicas, el EVA dirigido por un maestro puede ser la mejor opción, resaltando que, en este último caso, la aplicación de la herramienta tecnológica implica más trabajo para el docente, requiriendo además, cierta experiencia tecnológica, a diferencia de los EVA institucionales que facilitan esta labor.

Actualmente el empleo de las redes de aprendizaje se caracteriza por presentar un diseño educativo cuyo eje corresponde al trabajo realizado por medio de las plataformas de tele formación, instaladas en servidores que no necesitan el acceso a Internet para su funcionamiento, además que los materiales didácticos se encuentran fuera de línea y que pueden ser descargables, obtenerse bajo demanda y por medio de repositorios, utilizando objetos de aprendizaje caracterizados por ser de tipo mixto. Por tanto, la diversidad de contextos en que se desarrollan los procesos educativos exige adoptar e integrar el modelo pedagógico en cada caso a un EVA, de acuerdo con las condiciones reales desde el punto de vista tecnológico, organizacional y aplicando una estrategia didáctica que potencie el aprendizaje.

En concordancia con lo expresado por Bustos \& Coll (2010), la generación, análisis y comprensión de las configuraciones de los distintos entornos para la enseñanza y el aprendizaje virtual implica la necesidad de reconocer la enorme complejidad intrínseca, asociada al conjunto de usos de estas tecnologías, la diversidad y la heterogeneidad de criterios empleados para describir y clasificar estas herramientas, siendo, fundamentalmente, cinco los criterios que aparecen en las clasificaciones, bien sea de forma independiente o combinados, que son:

1. Relacionado con la configuración de los recursos tecnológicos empleados, entre los que se puede nombrar las computadoras, redes de computación, sistemas de interconexión, soporte y formato de la información, plataformas, sistemas de administración de aprendizaje o contenidos y aulas virtuales, entre algunos otros.

2. Según el uso de herramientas y aplicaciones que permiten combinar los recursos disponibles, como simulaciones, materiales multimedia, tableros electrónicos, correo electrónico, listas de correo, grupos de noticias, mensajería instantánea, videoconferencia interactiva, etc.

3. La mayor o menor amplitud y riqueza de las interacciones que las tecnologías seleccionadas posibilitan.

4. Tiene que ver con el carácter sincrónico o asincrónico de las interacciones. 
5. De acuerdo con las finalidades y objetivos educativos que se desean alcanzar y las concepciones implícitas o explícitas del aprendizaje y de la enseñanza en las que se sustentan.

En el caso de las ventajas que ofrecen los EVA para el desarrollo de los procesos de enseñanza-aprendizaje se puede mencionar:

- Accesibilidad: El aprendizaje a distancia alcanza cada vez más a una mayor cantidad de estudiantes, especialmente de áreas remotas del mundo, permitiéndoles acceder a todos los niveles de educación, siempre que posean una computadora, tableta o teléfono inteligente.

- Naturaleza inclusiva: A muchos estudiantes que generalmente son tímidos en las aulas físicas les resulta más fácil usar el software de aula virtual, permitiéndoles ser más expresivos y presentes.

- Mejor gestión del tiempo: En la mayoría de los casos, para los usuarios que estudian y trabajan, acceder a la instrucción académica por medio de un aula virtual interactiva resulta mucho más fácil que tomarse un tiempo todos los días para ir a un campus físico, esto permite administrar el tiempo de manera más efectiva, además el aprendizaje virtual, generalmente, puede tener lugar en el momento que sea más conveniente para el estudiante.

- Reducciones de costos: Esto se deriva de lo asequible que es el aprendizaje a distancia, ayudando, por una parte, a los estudiantes que no tienen que desplazarse, debido que no es necesario estar en un salón de clases a una hora o día específico, lo cual representa que no existen costos de viajes, y, por otra parte, a las instituciones que no tienen que expandirse constantemente y agregar aulas adicionales a su campus para albergar a más estudiantes. Asimismo, el costo de un software de videoconferencia es mucho menor que los costos operativos continuos que implica el mantenimiento de un aula física.

- Resultados instantáneos: Además de facilitar la gestión del aprendizaje para los estudiantes, el aprendizaje en línea también les proporciona resultados más rápidos en sus evaluaciones, por lo que no tienen que esperar semanas para obtener los puntajes de sus exámenes, debido que estos se califican tan pronto como los terminan, mediante la aplicación de un software de aula virtual (CIF Training, 2018).

Por otra parte, entre las desventajas más comunes de los entornos de aprendizaje virtual se puede mencionar:

- Falta de interacción social: El aspecto social de unirse a un colegio o universidad es una de las cosas más importantes en la vida de un estudiante, sin embargo, en muchos ejemplos de aulas virtuales, se observa cómo se elimina esta parte 
importante de la experiencia de la educación superior, encontrando que los estudiantes pueden aprender mucho desde sus hogares y colaborar con otros, pero no se acerca al nivel de socialización que pueden tener en el campus.

- Falta de disciplina: Puede ser muy complicado para los estudiantes desarrollar la disciplina requerida en la vida cuando saben que todo lo que necesitan hacer es encender la computadora y tomar una clase, además es fundamental que tengan la motivación necesaria para desarrollar el curso, siendo este tipo de aprendizaje más difícil para aquellos que son propensos a la procrastinación.

- Aprendizaje individual: En caso de necesitar una aclaración o explicación adicional acerca de un tema, esta respuesta no está disponible al instante como lo estaría en los cursos presenciales y aunque se puede solicitar una aclaración en una situación de aprendizaje virtual, la respuesta puede demorarse debido a la disponibilidad en línea del instructor o docente.

- Aprendizaje sin intervención: Quizás sea esto una de las mayores desventajas del aprendizaje virtual, debido que los estudiantes no tienen oportunidades de aprendizaje práctico, al no poder entrar en laboratorios, desarrollar experimentos o experimentar físicamente algo que es esencial para un sujeto, haciendo que las lecciones en línea a veces sean aburridas o inútiles.

- Problemas técnicos: Si bien es una de las principales ventajas del aprendizaje virtual que los estudiantes de áreas remotas puedan aprender lo que quieran, también tiene un lado negativo porque estos estudiantes a menudo enfrentan problemas tecnológicos, tal como que no tengan Internet estable o electricidad, haciendo que la experiencia educativa sea frustrante.

- Facilita el engaño: Una de las mayores desventajas cuando se trata del aprendizaje digital es que es muy difícil para los maestros evitar las trampas por parte de los estudiantes, bien sea por exámenes o tareas de clase, siendo muy complicado mantener un nivel de equidad debido que todos usan sus propias computadoras y están sentados en un entorno propio, presentándose casos en que los estudiantes le piden a otra persona que tome una prueba en su nombre a veces (CIF Training, 2018).

La educación a través del uso de herramientas tecnológicas adquiere una mayor fortaleza $\mathrm{y}$ ventaja si se fundamenta en un modelo de comportamiento, considerando que frente a un entorno virtual los estudiantes no tienen un modelo a seguir o imitar y, en consecuencia, deben ser y sentirse ellos mismos sin modificar ningún aspecto de su identidad como individuos, situación que genera que cada uno se forme de manera independiente sin necesidad de copiar rasgos o características de comportamiento del instructor o profesor (Moya et al., 2015).

Por tanto, el estudiante debe buscar por sí mismo los conocimientos que desea adquirir, especialmente en los niveles superiores de la educación, logrando esto con la aplicación 
de métodos investigativos, tomando como recursos los compañeros, la observación, experiencias personales y un proceso de reflexión, representando estos factores el mejor apoyo y garantía para llegar a participar de manera activa en los espacios de intercambio de ideas y conocimientos de los EVA, utilizando los diversos recursos tecnológicos disponibles.

Con respecto al docente, la perfecta integración de las TIC en la educación dependerá en gran medida de la habilidad de este para estructurar un efectivo ambiente de aprendizaje, de tal manera que es necesario que redefina esquemas tradicionales con un aprendizaje fundamentado en la cooperación y el trabajo en equipo; sin embargo, en mucha ocasiones aquellos docentes definidos como de la "vieja escuela" no entienden completamente el empleo y relación de las TIC con los procesos educativos, sin comprender que esta fórmula pueden generar un aprendizaje significativo, limitándola a simples herramientas que permiten acceder y transmitir información, criterio errado que sigue afectando a la educación tradicional (Granda et al., 2021).

\section{Resultados y Discusión}

La revisión de literatura permite deducir que la incorporación de las TIC a la educación por medio de los entornos virtuales de aprendizaje (EVA) ha revolucionado el proceso educativo, convirtiéndose en un proceso fundamental que desde la concepción conceptual va más allá de la simple aplicación de las herramientas tecnológicas que forman parte del sistema educativo, se refiere a una construcción didáctica y la forma cómo se construye y consolida un aprendizaje significativo basado en la tecnología, por lo que en un sentido estrictamente pedagógico se habla del uso tecnológico a la educación.

Por otra parte, los entornos virtuales en la educación poseen una estructura definida que se debe adaptar a las necesidades específicas de cada institución o nivel de estudios, por lo que para que el proceso enseñanza-aprendizaje funcione dentro del ambiente virtual es fundamental establecer una estrategia metodológica que responda a un diseño instruccional que potencie las cualidades operativas propuestas por una plataforma digital, para el diseño de eficientes módulos instruccionales en función de los resultados que se desean alcanzar.

Asimismo, por una parte, el dominio que poseen los estudiantes de las herramientas tecnológicas ha posibilitado que se masifique dentro de la educación, razón por la cual la mayor parte de las instituciones educativas han incursionado en esta área con resultados altamente positivos y, por otro lado, los espacios virtuales requieren de instructores o docentes capacitados y motivados que tengan la disposición de diseñar contenidos especializados que les permitan interactuar de manera permanente con los alumnos, por lo que el manejo correcto de las nuevas tecnologías implica no solamente el conocimiento 
de los medios disponibles, sino también el dominio de aquellos que resultan fundamentales y estratégicos para los procesos de formación.

\section{Conclusiones}

- Los EVA cumplen un papel innovador en la educación, constituyendo una opción extraordinaria para potenciar el proceso de enseñanza-aprendizaje, debido que pone a disposición espacios donde los estudiantes se interconectan entre sí y con el docente, permitiéndoles adquirir conocimientos nuevos que surgen del proceso de análisis y reflexión colaborativa, superando la posición de simples receptores del mensaje, además de ofrecer la posibilidad de diseñar estrategias que se establezcan como nuevos paradigmas de enseñanza.

- Asimismo, el empleo de las TIC como parte del proceso enseñanza-aprendizaje en el aula, requiere que los docentes adquieran un conjunto de competencias que les permita desarrollar una metodología con capacidad de aprovechar las herramientas tecnológicas disponibles, por tanto, la capacitación docente debe ser considerada una de las primeras opciones previo a afrontar los nuevos retos educativos.

- En consecuencia, las herramientas tecnológicas han incrementado el nivel de significancia y concepción educativa al establecer modelos novedosos de comunicación y generando espacios de formación, información, debate y reflexión, traspasando las barreras de los procesos educativos tradicionales, convirtiéndose en valiosos recursos para el aprendizaje y formación de estudiantes con elevados niveles de competencias personales y profesionales.

\section{Referencias Bibliográficas}

Granda, L. Y. G., Jaramillo, L. A. R., \& Macas, D. F. J. (2021). El docente y la alfabetización digital en la educación del siglo XXI. Sociedad \& Tecnología, 4(S2), 377-390. https://doi.org/10.51247/st.v4iS2.158

Barker, J., \& Gossman, P. (2013). The learning impact of a virtual learning environment: Students' views. Teacher Education Advancement Network Journal (TEAN), 5(2), 19-38.

Bustos Sánchez, A., \& Coll Salvador, C. (2010). Los entornos virtuales como espacios de enseñanza y aprendizaje. Una perspectiva psicoeducativa para su caracterización y análisis. Revista mexicana de investigación educativa, 15(44), 163-184. 
CIF Training. (2018, enero 22). The Advantages and Disadvantages of Virtual Learning. CIF Training website: https://ciftraining.ie/2018/01/advantages-disadvantagesvirtual-learning/

Dudeney, G., \& Hockly, N. (2007). How to Teach English with Technology. Pearson/Longman.

García-Aretio, L., Ruiz-Corbella, M., \& Figaredo, D. (2007). De la educación a distancia a la educación virtual.

Green, T. D., Brown, A., \& Robinson, L. (2008). Making the Most of the Web in Your Classroom: A Teacher's Guide to Blogs, Podcasts, Wikis, Pages, and Sites. Corwin Press.

Hernández, R. M. (2017). Impacto de las TIC en la educación: Retos y Perspectivas. $\begin{array}{llll}\text { Propósitos } & \text { y } \quad \text { Representaciones, 325-347. }\end{array}$ https://doi.org/10.20511/pyr2017.v5n1.149

Herrera Mosquera, L. (2017). Impact of Implementing a Virtual Learning Environment (VLE) in the EFL Classroom. Íkala, Revista de Lenguaje y Cultura, 22(3), 479498. https://doi.org/10.17533/udea.ikala.v22n03a07

Moscosio, M. Á. B. (2001). Redes de aprendizaje. Guía para la enseñanza y el aprendizaje en red. Revista https://revistascientificas.us.es/index.php/fuentes/article/view/2770

Moya, E. J. G., Llumiquinga-Quispe, S. del R., \& Ortiz-Remache, K. J. (2015). Caracterización de entornos virtuales de enseñanza aprendizaje (EVEA) en la educación virtual. Ciencias Holguín, XXI (4), 1-16.

Romero, E. C. (2019). Entornos virtuales de aprendizaje y su rol innovador en el proceso de enseñanza. ReHuSo: Revista de Ciencias Humanísticas y Sociales, 4(1), 119127.

Sánchez, A. B., \& Salvador, C. C. (2010). Los entornos virtuales como espacios de enseñanza y aprendizaje. 15(4), 23.

Underwood, J. (2009). The Impact of digital technology. Becta, 1(1), 1-27.

Vidal Ledo, M., Llanusa Ruiz, S., Diego Olite, F., \& Vialart Vidal, N. (2008). Entornos virtuales de enseñanza-aprendizaje. Educación Médica Superior, 22(1), 0-0.

Walker, A., \& White, G. (2013). Technology Enhanced Language Learning: Connecting theory and practice. OUP Oxford.

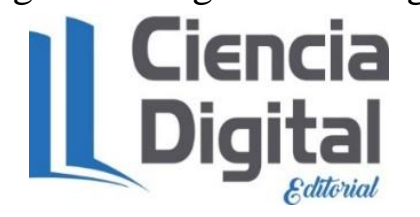


El artículo que se publica es de exclusiva responsabilidad de los autores y no necesariamente reflejan el pensamiento de la Revista Alfa Publicaciones.

\section{Ciencia}

El artículo queda en propiedad de la revista y, por tanto, su publicación parcial y/o total en otro medio tiene que ser autorizado por el director de la Revista Alfa Publicaciones.
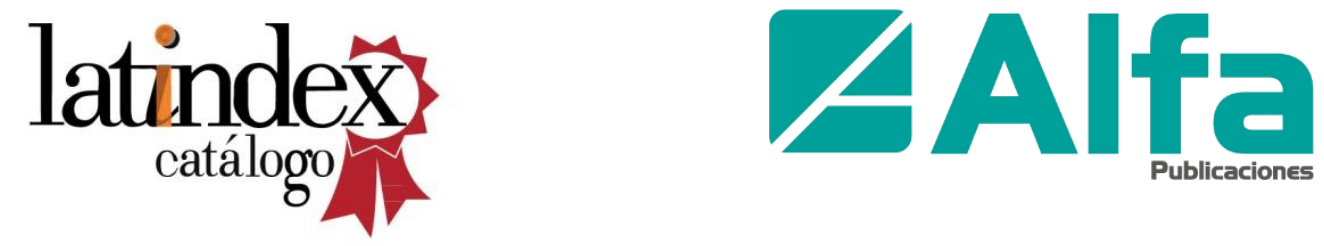

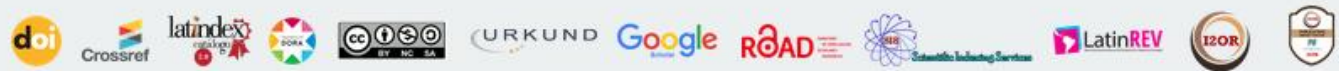

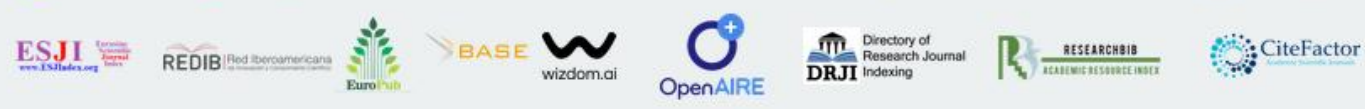

